\title{
Heart rate n-variability (HRnV) and its application to risk stratification of chest pain patients in the emergency department
}

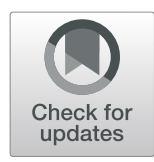

Nan Liu ${ }^{1,2^{*}}$, Dagang Guo ${ }^{3}$, Zhi Xiong Koh ${ }^{4}$, Andrew Fu Wah Ho ${ }^{1,3,5}$, Feng Xie ${ }^{1}$, Takashi Tagami ${ }^{6}$, Jeffrey Tadashi Sakamoto ${ }^{7}$, Pin Pin Pek ${ }^{1,4}$, Bibhas Chakraborty ${ }^{1}$, Swee Han Lim ${ }^{4}$, Jack Wei Chieh Tan ${ }^{8}$ and Marcus Eng Hock Ong ${ }^{1,2,4}$

\begin{abstract}
Background: Chest pain is one of the most common complaints among patients presenting to the emergency department (ED). Causes of chest pain can be benign or life threatening, making accurate risk stratification a critical issue in the ED. In addition to the use of established clinical scores, prior studies have attempted to create predictive models with heart rate variability (HRV). In this study, we proposed heart rate n-variability (HRnV), an alternative representation of beat-to-beat variation in electrocardiogram (ECG), and investigated its association with major adverse cardiac events (MACE) in ED patients with chest pain.

Methods: We conducted a retrospective analysis of data collected from the ED of a tertiary hospital in Singapore between September 2010 and July 2015. Patients $>20$ years old who presented to the ED with chief complaint of chest pain were conveniently recruited. Five to six-minute single-lead ECGs, demographics, medical history, troponin, and other required variables were collected. We developed the HRnV-Calc software to calculate HRnV parameters. The primary outcome was 30-day MACE, which included all-cause death, acute myocardial infarction, and revascularization. Univariable and multivariable logistic regression analyses were conducted to investigate the association between individual risk factors and the outcome. Receiver operating characteristic (ROC) analysis was performed to compare the HRnV model (based on leave-one-out cross-validation) against other clinical scores in predicting 30-day MACE.

(Continued on next page)
\end{abstract}

\footnotetext{
* Correspondence: liu.nan@duke-nus.edu.sg

'Duke-NUS Medical School, National University of Singapore, 8 College Road,

Singapore 169857, Singapore

${ }^{2}$ Health Services Research Centre, Singapore Health Services, 20 College

Road, Singapore 169856, Singapore

Full list of author information is available at the end of the article
} 


\begin{abstract}
(Continued from previous page)
Results: A total of 795 patients were included in the analysis, of which 247 (31\%) had MACE within 30 days. The MACE group was older, with a higher proportion being male patients. Twenty-one conventional HRV and $115 \mathrm{HRnV}$ parameters were calculated. In univariable analysis, eleven HRV and $48 \mathrm{HRnV}$ parameters were significantly associated with 30-day MACE. The multivariable stepwise logistic regression identified 16 predictors that were strongly associated with MACE outcome; these predictors consisted of one HRV, seven HRnV parameters, troponin, ST segment changes, and several other factors. The HRnV model outperformed several clinical scores in the ROC analysis.
\end{abstract}

Conclusions: The novel HRnV representation demonstrated its value of augmenting HRV and traditional risk factors in designing a robust risk stratification tool for patients with chest pain in the ED.

Keywords: Heart rate variability (HRV), Heart rate n-variability (HRnV), Electrocardiogram, Chest pain, Risk stratification, Emergency department

\section{Background}

Chest pain, which may be caused by life-threatening myocardial infarction (MI) or benign musculoskeletal pain, is one of the most common presenting complaints in the emergency department (ED) [1-3]. Majority of chest pain patients are subjected to extensive diagnostic tests to rule out acute coronary syndrome (ACS), resulting in oftentimes, prolonged and costly ED admission, with only a small proportion of these patients eventually receiving a diagnosis of ACS [3]. This can strain crowded EDs and reduce availability of resources for patients who need urgent medical attention. Hence, early identification of chest pain patients who are at high-risk of developing adverse cardiac events has been a pressing issue to contend with in the ED. Several established clinical scores have been used for risk stratifying chest pain patients in the ED $[4,5]$, including the History, ECG, Age, Risk factors and Troponin (HEART) [6], the Thrombolysis in Myocardial Infarction (TIMI) [7], and the Global Registry of Acute Coronary Events (GRACE) [8] scores. Of these scores, the HEART score is the most accurate and widely used [5,9-12], with recent studies focusing on the development of risk score-based clinical pathways for rapid, yet safe discharge of low-risk patients $[1,3,13,14]$.

In a recent review of clinical scores for ED patients with chest pain [5], heart rate variability (HRV) has demonstrated its capability in building predictive models for accurate risk stratification [15-17]. HRV is a widely adopted tool for evaluating changes in cardiac autonomic regulation, and has been shown to be strongly associated with the autonomic nervous system (ANS) [18-20]. HRV analysis characterizes the beat-to-beat variation in an electrocardiogram (ECG) by utilizing time and frequency domains, and nonlinear analyses [19]. Reduced HRV has been found to be a significant predictor of adverse cardiac outcomes [21]. Given the complexity of quantifying HRV representation, several tools such as the PhysioNet
Cardiovascular Signal Toolbox [22] and Kubios HRV [23] have been developed to standardize HRV analyses.

Based on the principle of parameter calculation on normal R-R intervals (RRIs; in this paper, RRIs are equivalent to normal-to-normal $[\mathrm{NN}]$ intervals, in which abnormal beats have been removed), HRV analysis generates only one set of parameters from a fixed length of ECG record. This limits the amount of information that can be extracted from raw ECG signals. In this paper, we proposed a novel representation of beat-to-beat variation, named as heart rate n-variability (HRnV) [24] to characterize RRIs from a different perspective. With the use of HRnV measures, multiple sets of parameters can be calculated from the same ECG record, which significantly increases the amount of extracted information. Our study is the first clinical application and evaluation of the HRnV representation in risk stratification of chest pain patients in the ED. We hypothesized that HRnV, while closely related to conventional HRV, can provide supplementary information associated with adverse cardiac events. We also investigated the potential use of $\mathrm{HRnV}$ parameters to develop risk prediction tools.

\section{Methods \\ Study design and setting}

We conducted a retrospective analysis of data collected in our previous study on risk stratification of chest pain patients in the ED [9]. A convenience sample of patients was recruited at the ED of Singapore General Hospital, a tertiary hospital with around-the-clock primary percutaneous coronary intervention capabilities and a median door-to-balloon time of $101 \mathrm{~min}$ [25], between September 2010 and July 2015. At ED triage, patients are classified using the Patient Acuity Category Scale (PACS), with PACS 1 patients being the most critically ill and requiring immediate medical attention and PACS 4 patients being non-urgent cases. In this study, patients > 20 years old who presented to the ED with chief 
complaint of chest pain and with PACS of 1 or 2 were included. Patients were excluded from the study if they had ST-elevation myocardial infarction (STEMI) or an obvious non-cardiac etiology of chest pain diagnosed by the primary emergency physician. Patients were also excluded if their ECGs had high level of noise or if they were in non-sinus rhythm; these criteria were applied to ensure the quality of HRV and HRnV analyses. Ethical approval was obtained from the Centralized Institutional Review Board (CIRB, Ref: 2014/584/C) of SingHealth, the largest public healthcare system in Singapore that includes the Singapore General Hospital as a key partner. Patient consent was waived for this study.

\section{Data collection}

During the data collection period, five to six-minute single-lead (lead II) ECG recordings were retrieved from the X-Series Monitor (ZOLL Medical Corporation, Chelmsford, MA). The first set of vital signs and troponin values from the recruited patients were extracted from the hospital's electronic health records (EHR). In this study, high-sensitivity troponin- $\mathrm{T}$ was used, and an abnormal value was defined as $>0.03 \mathrm{ng} / \mathrm{mL}$ [26]; it was further stratified into three groups and coded as 0 if the value was $\leq 0.03 \mathrm{ng} / \mathrm{mL}, 1$ if the value was between 1 and 3 times the normal limit, and 2 if the value was $>3$ times the normal limit. Additionally, patients' first 12-lead ECGs were interpreted by two independent clinical reviewers. Pathologic ST-elevation, ST-depression, T-wave inversions, and Q-waves were recorded. Patient demographics, medical history, and information required for computing the HEART, TIMI, and GRACE scores were retrospectively reviewed and obtained from EHR.

\section{Proposed HRnV representation of beat-to-beat variation in ECG \\ $H R_{n} V$ : a novel measure with non-overlapping RRIs}

Prior to introducing the new $\mathrm{HR}_{n} \mathrm{~V}$ measure, we define a new type of RRI called $\mathrm{RR}_{n} \mathrm{I}$, where $1 \leq n \leq N$, and $N \ll \hat{N}$; $\hat{N}$ is the total number of RRIs. The definition of $R_{n} I$ is illustrated in Fig. 1a. When $n=1, \mathrm{RR}_{n} \mathrm{I}$ is equivalent to conventional RRI. When $n>1$, every $n$ adjacent RRI is connected to form a new sequence of $\mathrm{RR}_{n} \mathrm{Is}$. By using
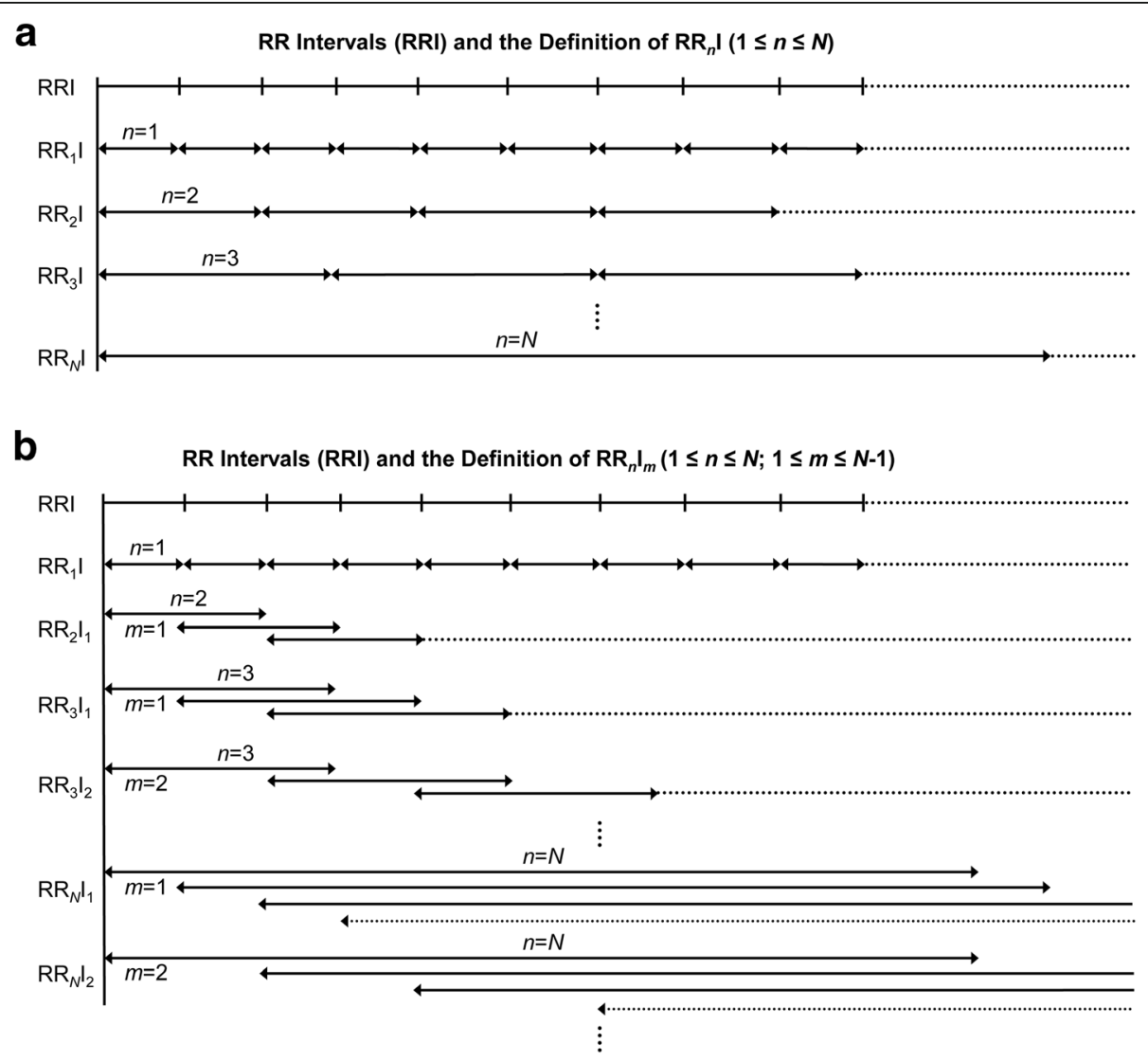

Fig. 1 a Illustration of R-R intervals (RRIs) and the definition of $R R_{n} I$ where $1 \leq n \leq N$ and $N \ll \hat{N}$. $\hat{N}$ is the total number of RRIs; b IIlustration of RRIs and the definition of $R_{n} I_{m}$ where $1 \leq n \leq N, 1 \leq m \leq N-1$, and $N \ll \hat{N}$. $\hat{N}$ is the total number of RRIs and $m$ indicates the non-overlapping portion between two consecutive $R_{n} I_{m}$ sequences 
this strategy, we can create a maximum number of $(N-1)$ new $\mathrm{RR}_{n} \mathrm{I}$ sequences from conventional single RRI sequence. With these newly generated $R_{n} I$ sequences, the calculation of $\mathrm{HR}_{n} \mathrm{~V}$ parameters is straightforward and can be accomplished by applying established quantitative methods including time and frequency domain analyses and nonlinear analysis [18, 19]. In describing this new measure, we use the term " $\mathrm{HR}_{n} \mathrm{~V}$ " prior to parameter names to indicate that these parameters are calculated from $\mathrm{RR}_{n} \mathrm{I}$ sequences. As noted in the above, $\mathrm{HR}_{n} \mathrm{~V}$ is a novel measure based on newly generated, non-overlapping $\mathrm{RR}_{n} \mathrm{Is}$. The computed $\mathrm{HR}_{n} \mathrm{~V}$ parameters include but are not limited to the following: the average of $\mathrm{RR}_{n} \mathrm{Is}\left(\mathrm{HR}_{n} \mathrm{~V}\right.$ mean $\mathrm{NN})$, standard deviation of $\mathrm{RR}_{n} \mathrm{Is}\left(\mathrm{HR}_{n} \mathrm{~V} \mathrm{SDNN}\right)$, square root of the mean squared differences between $\mathrm{RR}_{n} \mathrm{Is}\left(\mathrm{HR}_{n} \mathrm{~V}\right.$ RMSSD), the number of times that the absolute difference between two successive $\mathrm{RR}_{n} \mathrm{Is}$ exceeds $50 \mathrm{~ms} \quad\left(\mathrm{HR}_{n} \mathrm{~V}\right.$ NN50), $\mathrm{HR}_{n} \mathrm{~V}$ NN50 divided by the total number of $\mathrm{RR}_{n}$ Is $\left(\mathrm{HR}_{n} \mathrm{~V}\right.$ pNN50), the integral of the $\mathrm{RR}_{n} \mathrm{I}$ histogram divided by the height of the histogram $\left(\mathrm{HR}_{n} \mathrm{~V}\right.$ triangular index), low frequency power $\left(\mathrm{HR}_{n} \mathrm{~V}\right.$ LF power), high frequency power $\left(\mathrm{HR}_{n} \mathrm{~V}\right.$ HF power), approximate entropy $\left(\mathrm{HR}_{n} \mathrm{~V} \mathrm{ApEn}\right)$, sample entropy $\left(\mathrm{HR}_{n} \mathrm{~V}\right.$ SampEn), and detrended fluctuation analysis $\left(\mathrm{HR}_{n} \mathrm{~V} \mathrm{DFA}\right)$, among others. Notably, two new parameters $\mathrm{NN50} n$ and $\mathrm{pNN} 50 n$ are created, where $50 \times n$ $\mathrm{ms}$ is set as the threshold to assess the difference between pairs of consecutive $\mathrm{RR}_{n} \mathrm{Is}$.

\section{$H R_{n} V_{m}$ : a novel measure with overlapping RRIs}

Like $\mathrm{RR}_{n} \mathrm{I}$ that is used in $\mathrm{HR}_{n} \mathrm{~V}$, to define $\mathrm{HR}_{n} \mathrm{~V}_{m}$ measure, we introduce another type of RRI called $\mathrm{RR}_{n} \mathrm{I}_{m}$, where $1 \leq$ $n \leq N, 1 \leq m \leq N-1$, and $N \ll \hat{N}$. In the $\mathrm{RR}_{n} \mathrm{I}_{m}$ sequence, $m$ is used to indicate the level of overlap between consecutive $\mathrm{RR}_{n} \mathrm{I}_{m}$ sequences. As illustrated in Fig. $1 \mathrm{~b},(n-m)$ RRIs form the overlapping portions. When $m=n, R_{n} I_{m}$ becomes $\mathrm{RR}_{n} \mathrm{I}$; therefore, the upper limit of $m$ is $N-1$. By controlling the overlap among these newly generated $\mathrm{RR}_{n} \mathrm{I}_{m}$ sequences, we can create a maximum number of $(N \times(N-1) / 2) R R_{n} I_{m}$ sequences (excluding the $\mathrm{RR}_{n} \mathrm{I}$ sequence) from conventional single RRI sequence. For each of the newly created $R R_{n} I_{m}$ sequences, we apply time and frequency domain analyses, and nonlinear analysis to calculate $\mathrm{HR}_{n} \mathrm{~V}_{m}$ parameters. We add the term $" \mathrm{HR}_{n} \mathrm{~V}_{m}$ " prior to the parameters to denote that they are computed from $\mathrm{RR}_{n} \mathrm{I}_{m}$ sequences. For example, the average $\mathrm{RR}_{n} \mathrm{I}_{m}$ intervals and the sample entropy are written as $\mathrm{HR}_{n} \mathrm{~V}_{m}$ mean $\mathrm{NN}$ and $\mathrm{HR}_{n} \mathrm{~V}_{m}$ SampEn, respectively. The $\mathrm{HR}_{n} \mathrm{~V}_{m}$ measure extracts more information than $\mathrm{HR}_{n} \mathrm{~V}$, by adopting a strategy of controlling sequence overlap.

\section{$\mathrm{HRnV}$ analysis and parameter calculation}

We developed the HRnV-Calc software suite (https:// github.com/nliulab/HRnV) to calculate $\mathrm{HRnV}$ parameters.
The HRnV-Calc software integrates functions from the PhysioNet Cardiovascular Signal Toolbox [22] to perform standardized ECG signal processing and QRS complex detection. Given the short ECG records in this study, the upper limit of $n$ was set as three; thus, six sets of parameters were calculated, namely $\mathrm{HRV}, \mathrm{HR}_{2} \mathrm{~V}, \mathrm{HR}_{2} \mathrm{~V}_{1}, \mathrm{HR}_{3} \mathrm{~V}$, $\mathrm{HR}_{3} \mathrm{~V}_{1}$, and $\mathrm{HR}_{3} \mathrm{~V}_{2}$.

\section{Clinical outcomes}

The primary endpoint in this study was a composite outcome of major adverse cardiac events (MACE) [27], including all-cause death, acute myocardial infarction (AMI), and revascularization (coronary artery bypass graft [CABG] or percutaneous coronary intervention $[\mathrm{PCI}])$ within 30 days of ED presentation.

\section{Statistical analysis}

Continuous variables were presented as mean and standard deviation and compared between two categories of the primary outcome (MACE) using two-sample t-test. Categorical variables were presented as frequency and percentage and compared between two categories of the primary outcome (MACE) using chi-square test. A statistically significant difference was defined as $p<0.05$. To evaluate the HRnV parameters and other risk factors, we conducted univariable and multivariable analyses and subsequently developed simple prediction models using traditional logistic regression. In building the $\mathrm{HRnV}$ prediction model, we selected candidate variables with $p<$ 0.2 in the univariable analysis and fed them into the multivariable stepwise logistic regression. To evaluate the predictive performance, we used leave-one-out cross-validation (LOOCV) to conduct the analysis.

Receiver operating characteristic (ROC) analysis [28] was performed to compare prediction performances among the HRnV model, HEART, TIMI and GRACE scores. The area under the ROC curve (AUC), sensitivity, specificity, positive predictive value (PPV), and negative predictive value (NPV) were reported as predictive measures. Data preparation, descriptive analysis, and predictive model development were performed in $\mathrm{R}$ version 3.6.0 (R Foundation, Vienna, Austria); ROC analysis was conducted in MATLAB R2019a (MathWorks, Natick, MA).

\section{Results}

A total of 795 patients were selected from the originally recruited 922 patients [9]. Twenty-eight patients were excluded for ECG recording issues, four were excluded for obvious non-cardiac chest pain, and 95 were excluded for irregular rhythm/artifacts. Among the included 795 patients, 247 (31\%) had the primary outcome of 30-day MACE. Table 1 shows patient baseline characteristics. Patients with the primary outcome were older 
Table 1 Patient baseline characteristics

\begin{tabular}{|c|c|c|c|c|}
\hline & Total $(\boldsymbol{n}=795)$ & $\operatorname{MACE}(\boldsymbol{n}=247)$ & Non-MACE $(\boldsymbol{n}=548)$ & $\boldsymbol{p}$-value \\
\hline Age, mean (SD) & $59.63(12.88)$ & $61.06(11.38)$ & $58.99(13.47)$ & 0.035 \\
\hline Male gender, n (\%) & $542(68.2)$ & $188(76.1)$ & $354(64.6)$ & 0.002 \\
\hline Race, n (\%) & & & & 0.623 \\
\hline Chinese & $492(61.9)$ & $159(64.4)$ & $333(60.8)$ & \\
\hline Indian & $129(16.2)$ & $34(13.8)$ & $95(17.3)$ & \\
\hline Malay & $150(18.9)$ & 46 (18.6) & $104(19.0)$ & \\
\hline Other & $24(3.0)$ & $8(3.2)$ & $16(2.9)$ & \\
\hline \multicolumn{5}{|l|}{ Medical history, n (\%) } \\
\hline Ischemic heart disease & $343(43.1)$ & $115(46.6)$ & $228(41.6)$ & 0.22 \\
\hline Diabetes & $278(35.0)$ & $106(42.9)$ & $172(31.4)$ & 0.002 \\
\hline Hypertension & $509(64.0)$ & $161(65.2)$ & $348(63.5)$ & 0.707 \\
\hline Hypercholesterolemia & $476(59.9)$ & $151(61.1)$ & $325(59.3)$ & 0.683 \\
\hline Stroke & $58(7.3)$ & $15(6.1)$ & $43(7.8)$ & 0.458 \\
\hline Cancer & $29(3.6)$ & $7(2.8)$ & $22(4.0)$ & 0.537 \\
\hline Respiratory disease & $31(3.9)$ & $5(2.0)$ & $26(4.7)$ & 0.102 \\
\hline Chronic kidney disease & $87(10.9)$ & $26(10.5)$ & $61(11.1)$ & 0.32 \\
\hline Congestive heart failure & $38(4.8)$ & $9(3.6)$ & $29(5.3)$ & 0.407 \\
\hline History of $\mathrm{PCl}$ & $199(25.0)$ & $68(27.5)$ & 131 (23.9) & 0.316 \\
\hline History of CABG & $71(8.9)$ & $26(10.5)$ & $45(8.2)$ & 0.355 \\
\hline History of AMI & $133(16.7)$ & 48 (19.4) & 85 (15.5) & 0.288 \\
\hline Active smoker & $197(24.8)$ & 73 (29.6) & $124(22.6)$ & 0.003 \\
\hline
\end{tabular}

MACE Major adverse cardiac events, SD Standard deviation, $P C I$ Percutaneous coronary intervention, CABG Coronary artery bypass graft, $A M I$ Acute myocardial infarction

(mean age 61 years vs. 59 years, $p=0.035$ ), with a higher proportion being males $(76.1 \%$ vs. $64.6 \%, p=0.002)$. There was no statistically significant difference between MACE and non-MACE groups in terms of patient ethnicity. Factors such as history of diabetes and current smoking status were significantly more prevalent in the group with MACE.

Descriptive analyses of HRV and HRnV parameters are tabulated in Table 2 . In this clinical case study, $N$ was set as 3, thus $\mathrm{HR}_{2} \mathrm{~V}, \mathrm{HR}_{2} \mathrm{~V}_{1}, \mathrm{HR}_{3} \mathrm{~V}, \mathrm{HR}_{3} \mathrm{~V}_{1}$ and $\mathrm{HR}_{3} \mathrm{~V}_{2}$ parameters were calculated. Among time domain parameters such as mean NN, SDNN and RMSSD, the $\mathrm{HR}_{n} \mathrm{~V}$ and $\mathrm{HR}_{n} \mathrm{~V}_{m}$ values were generally incremental with an increase in $n$. Notably, $\mathrm{HR}_{2} \mathrm{~V}$ NN50 and $\mathrm{HR}_{3} \mathrm{~V}$ NN50 were much lower than conventional HRV NN50. Moreover, NN50n and pNN50n are parameters specifically applicable to the $\mathrm{HRnV}$ representation. Like time domain parameters, the same trend of changes in frequency domain parameters were observed. The magnitude of increment in VLF power and LF power was larger than that of HF power with increasing $n$. One exception, however, was the normalized HF power, where $\mathrm{HR}_{n} \mathrm{~V}$ and $\mathrm{HR}_{n} \mathrm{~V}_{m}$ parameters were smaller than that of HRV. In nonlinear analysis, there were marked differences in Poincaré SD2 values between HRV and HRnV parameters. $\mathrm{HR}_{2} \mathrm{~V}$ SampEn and $\mathrm{HR}_{3} \mathrm{~V}$ SampEn were considerably larger compared to SampEn parameters of $\mathrm{HRV}, \mathrm{HR}_{2} \mathrm{~V}_{1}, \mathrm{HR}_{3} \mathrm{~V}_{1}$, and $\mathrm{HR}_{3} \mathrm{~V}_{2}$, as their confidence intervals (CIs) were wide. The wide CI was due to insufficient data points of less than 200 [19], as our ECG recordings were only five to six minutes long. $H_{2} \mathrm{~V}_{1}$, $\mathrm{HR}_{3} \mathrm{~V}_{1}$ and $\mathrm{HR}_{3} \mathrm{~V}_{2}$ were free from this issue as they were calculated from overlapping $\mathrm{RR}_{n} \mathrm{I}_{m}$ sequences where more data points were available.

Table 3 presents the results of univariable analyses of $\mathrm{HR}_{n} \mathrm{~V}$ and $\mathrm{HR}_{n} \mathrm{~V}_{m}$ parameters. Eleven out of 21 conventional HRV parameters were statistically significant. Additionally, $13 \mathrm{HR}_{2} \mathrm{~V}$, six $\mathrm{HR}_{3} \mathrm{~V}, 11 \mathrm{HR}_{2} \mathrm{~V}_{1}$, seven $\mathrm{HR}_{3} \mathrm{~V}_{1}$ and $11 \mathrm{HR}_{3} \mathrm{~V}_{2}$ parameters were also significant. Overall, additional $115 \mathrm{HRnV}$ parameters were derived, among which 48 showed statistical significances between patients with 30-day MACE and those without. Among all HRV and HRnV parameters, mean NN, SDNN, RMSSD, NN50, pNN50, HF power, Poincaré SD1 and SD2 were statistically significant in at least five out of six measures (i.e., $\mathrm{HRV}, \mathrm{HR}_{2} \mathrm{~V}, \mathrm{HR}_{2} \mathrm{~V}_{1}, \mathrm{HR}_{3} \mathrm{~V}, \mathrm{HR}_{3} \mathrm{~V}_{1}$, and $\mathrm{HR}_{3} \mathrm{~V}_{2}$ ). Furthermore, skewness, LF power, SampEn, and ApEn, which did not demonstrate statistical significance in conventional HRV analysis, were statistically significant in $\mathrm{HRnV}$ representation. Table 4 presents the 
Table 2 Descriptive analyses of heart rate variability (HRV) and heart rate n-variability (HRnV) parameters

\begin{tabular}{|c|c|c|c|c|c|c|}
\hline & HRV & $\mathrm{HR}_{\mathbf{2}} \mathrm{V}$ & $\mathrm{HR}_{2} \mathrm{~V}_{1}$ & $\mathrm{HR}_{3} \mathrm{~V}$ & $\mathrm{HR}_{3} \mathrm{~V}_{1}$ & $\mathrm{HR}_{3} \mathrm{~V}_{2}$ \\
\hline Mean NN (s) & $829.40(169.49)$ & 1656.65 (339.85) & 1658.81 (338.99) & 2484.80 (509.33) & $2488.22(508.50)$ & $2485.02(509.84)$ \\
\hline SDNN (s) & $38.16(25.49)$ & $62.28(45.45)$ & $68.81(47.00)$ & $82.06(62.47)$ & $97.79(67.46)$ & $87.77(64.52)$ \\
\hline RMSSD (s) & $30.04(23.07)$ & $32.61(26.68)$ & $33.79(25.67)$ & $34.83(28.86)$ & $36.27(26.50)$ & $34.98(27.43)$ \\
\hline Skewness & $-0.65(2.34)$ & $-0.41(1.66)$ & $-0.59(1.95)$ & $-0.29(1.29)$ & $-0.55(1.69)$ & $-0.38(1.42)$ \\
\hline Kurtosis & $14.59(26.83)$ & 7.33 (13.58) & $10.17(17.90)$ & $5.15(8.13)$ & $8.06(12.92)$ & $5.98(9.75)$ \\
\hline Triangular index & $7.68(4.19)$ & $10.38(5.10)$ & $12.60(6.45)$ & $11.47(5.29)$ & $16.25(7.94)$ & $13.06(6.04)$ \\
\hline NN50 (count) & $21.08(33.98)$ & $14.46(20.35)$ & $29.35(40.03)$ & $11.57(15.05)$ & $35.29(44.34)$ & $17.41(22.51)$ \\
\hline pNN50 (\%) & $6.31(11.08)$ & $8.66(13.18)$ & $8.75(12.97)$ & $10.31(14.27)$ & $10.38(13.95)$ & $10.28(14.20)$ \\
\hline NN50n (count) & - & $4.16(9.72)$ & 8.45 (18.76) & $1.37(3.72)$ & $4.37(10.72)$ & $2.08(5.48)$ \\
\hline pNN50n (\%) & - & $2.60(6.67)$ & $2.64(6.47)$ & $1.32(3.95)$ & $1.39(3.86)$ & $1.33(3.87)$ \\
\hline Total power $\left(\mathrm{ms}^{2}\right)$ & $2518.30(4797.05)$ & $7797.46(16,947.44)$ & $9156.26(17,970.75)$ & $13,904.78(37,182.24)$ & $18,714.67(37,620.26)$ & $15,706.11(34,845.52)$ \\
\hline VLF power $\left(\mathrm{ms}^{2}\right)$ & 985.18 (1991.52) & $3401.42(6569.37)$ & 3922.74 (7987.46) & $6503.53(14,205.11)$ & $8772.26(17,986.63)$ & $7567.79(14,666.32)$ \\
\hline LF power $\left(\mathrm{ms}^{2}\right)$ & 732.36 (1841.88) & 2626.83 (7593.16) & $2782.48(7212.62)$ & $5091.49(18,402.20)$ & $5740.99(15,243.38)$ & $5397.76(16,001.18)$ \\
\hline HF power $\left(\mathrm{ms}^{2}\right)$ & 527.27 (1232.69) & 1328.86 (4033.96) & 1361.53 (3433.55) & 1661.69 (7237.55) & $1762.45(4851.11)$ & $1761.05(6477.63)$ \\
\hline LF power norm (nu) & $56.76(19.20)$ & $66.82(18.17)$ & $66.42(17.35)$ & $76.53(15.32)$ & $77.65(14.55)$ & $77.93(14.95)$ \\
\hline HF power norm (nu) & $43.24(19.20)$ & $33.18(18.17)$ & $33.58(17.35)$ & $23.47(15.32)$ & $22.35(14.55)$ & $22.07(14.95)$ \\
\hline LF/HF & $1.99(1.93)$ & $3.24(2.95)$ & $3.04(2.73)$ & $5.60(5.21)$ & $5.79(4.99)$ & $6.06(5.18)$ \\
\hline Poincaré SD1 (ms) & $21.27(16.34)$ & $23.12(18.93)$ & $23.92(18.18)$ & $24.72(20.50)$ & $25.68(18.77)$ & $24.80(19.46)$ \\
\hline Poincaré SD2 (ms) & $48.82(33.29)$ & $84.47(62.15)$ & $93.88(64.58)$ & 112.87 (86.62) & 135.55 (94.02) & $121.20(89.72)$ \\
\hline SampEn & $1.57(0.51)$ & $83.84(2324.24)$ & $1.33(0.48)$ & 248.48 (4020.64) & $1.06(0.41)$ & $1.14(0.45)$ \\
\hline ApEn & $0.99(0.20)$ & $0.72(0.18)$ & $0.91(0.17)$ & $0.60(0.15)$ & $0.84(0.17)$ & $0.70(0.15)$ \\
\hline DFA, a1 & $0.99(0.31)$ & $1.24(0.29)$ & $1.23(0.27)$ & $1.41(0.27)$ & $1.42(0.23)$ & $1.42(0.25)$ \\
\hline DFA, a2 & $0.95(0.22)$ & $0.98(0.35)$ & $0.98(0.22)$ & $0.86(0.65)$ & $1.01(0.22)$ & $1.02(0.36)$ \\
\hline
\end{tabular}

HRV Heart rate variability, mean NN Average of R-R intervals, SDNN Standard deviation of R-R intervals, RMSSD Square root of the mean squared differences between R-R intervals; NN50, the number of times that the absolute difference between 2 successive R-R intervals exceeds 50 ms; pNN50, NN50 divided by the total number of R-R intervals; NN50n, the number of times that the absolute difference between 2 successive RR $R_{n} / R_{n} l_{m}$ sequences exceeds $50 \times n$ ms; pNN50n, NN50n divided by the total number of $\mathrm{RR}_{n} l / \mathrm{RR}_{n} \mathrm{I}_{m}$ sequences; VLF Very low frequency, LF Low frequency, HF High frequency, SD Standard deviation, SampEn Sample entropy, ApEn Approximate entropy, DFA Detrended fluctuation analysis

results of the multivariable analyses of $\mathrm{HR}_{n} \mathrm{~V}$ and $\mathrm{HR}_{n} \mathrm{~V}_{m}$ parameters by adjusting for age and sex. After adjustment, several parameters such as NN50 of $\mathrm{HR}_{3} \mathrm{~V}$ and $\mathrm{HR}_{3} \mathrm{~V}_{2}$, and triangular index of $\mathrm{HRV}, \mathrm{HR}_{2} \mathrm{~V}$, and $\mathrm{HR}_{3} \mathrm{~V}_{2}$, became statistically non-significant, while parameters such as ApEn of $\mathrm{HR}_{2} \mathrm{~V}, \mathrm{HR}_{2} \mathrm{~V}_{1}$, and $\mathrm{HR}_{3} \mathrm{~V}_{2}$ became statistically significant.

Table 5 lists the 16 variables that were selected through multivariable stepwise logistic regression, among which there were one conventional HRV parameter and seven $\mathrm{HRnV}$ parameters. In addition to traditional predictors of adverse cardiac outcomes such as ST segment changes and troponin, $\mathrm{HR}_{2} \mathrm{~V}$ ApEn (OR = 0.095; 95\% CI 0.014-0.628), $\mathrm{HR}_{2} \mathrm{~V}_{1}$ ApEn (OR = 19.700; 95\% CI 2.942-131.900) and $\mathrm{HR}_{3} \mathrm{~V}$ skewness (1.560; 95\% CI 1.116-2.181) also demonstrated strong predictive power in assessing the risk of 30-day MACE. Figure 2 shows the ROC curves and Table 6 presents the results of ROC analysis in evaluating the predictive performance of the HRnV model (based on LOOCV), HEART, TIMI, and GRACE scores. The HRnV model achieved the highest AUC value and outperformed HEART, TIMI, and GRACE scores in terms of specificity, PPV, and NPV at the optimal cut-off scores, defined as the points nearest to the upper-left corner of the ROC curves.

\section{Discussion}

HRV has generated significant research interest in the past decades $[18,19,29]$, with majority of studies focusing on development of advanced nonlinear techniques to derive novel parameters $[30,31]$. There is, however, a paucity of research on alternative approaches to analyze RRIs. Vollmer [32] used relative RRIs to describe the relative variation of consecutive RRIs, with which HRV was analyzed. Likewise, we proposed a novel HRnV representation, providing more $\mathrm{HRnV}$ parameters than conventional HRV analysis. In this paper, we introduced two measures of $\mathrm{HRnV}$, namely $\mathrm{HR}_{n} \mathrm{~V}$ and $\mathrm{HR}_{n} \mathrm{~V}_{m}$. $\mathrm{HR}_{n} \mathrm{~V}$ was calculated based on non-overlapping $\mathrm{RR}_{n} \mathrm{I}$ sequences, while $\mathrm{HR}_{n} \mathrm{~V}_{m}$ was computed from overlapping 
Table 3 Univariable analysis of $H R_{n} V$ and $H R_{n} V_{m}$ parameters

\begin{tabular}{|c|c|c|c|c|c|c|}
\hline & HRV & & $\mathrm{HR}_{2} \mathrm{~V}$ & & $\mathrm{HR}_{3} \mathrm{~V}$ & \\
\hline & OR $(95 \% \mathrm{Cl})$ & $p$ & OR $(95 \% \mathrm{Cl})$ & $p$ & OR $(95 \% \mathrm{Cl})$ & $p$ \\
\hline Mean NN & $0.999(0.998-1.000)$ & $0.023^{*}$ & $0.999(0.999-1.000)$ & $0.023^{*}$ & $1.000(0.999-1.000)$ & $0.023^{*}$ \\
\hline SDNN & $0.992(0.986-0.999)$ & $0.023^{*}$ & $0.996(0.992-1.000)$ & $0.028^{*}$ & $0.997(0.995-1.000)$ & 0.060 \\
\hline RMSSD & $0.990(0.982-0.998)$ & $0.010^{*}$ & $0.992(0.985-0.998)$ & $0.011^{*}$ & 0.994 (0.988-0.999) & $0.030^{*}$ \\
\hline Skewness & $1.059(0.991-1.132)$ & 0.088 & $1.079(0.981-1.186)$ & 0.118 & $1.139(1.006-1.290)$ & $0.040^{*}$ \\
\hline Kurtosis & $1.006(1.000-1.011)$ & $0.038^{*}$ & 1.009 (0.998-1.019) & 0.113 & $1.011(0.993-1.029)$ & 0.242 \\
\hline Triangular index & $0.961(0.925-0.998)$ & $0.039^{*}$ & $0.967(0.938-0.997)$ & $0.032^{*}$ & $0.978(0.950-1.007)$ & 0.133 \\
\hline NN50 & $0.993(0.987-0.998)$ & $0.008^{*}$ & $0.989(0.981-0.998)$ & $0.012^{*}$ & $0.988(0.977-0.999)$ & $0.031^{*}$ \\
\hline pNN50 & $0.978(0.962-0.995)$ & $0.009^{*}$ & 0.984 (0.971-0.997) & $0.014^{*}$ & 0.987 (0.976-0.999) & $0.027^{*}$ \\
\hline NN50n & - & - & $0.982(0.964-1.001)$ & 0.065 & $0.952(0.905-1.002)$ & 0.059 \\
\hline pNN50n & - & - & $0.974(0.946-1.002)$ & 0.069 & $0.951(0.903-1.001)$ & 0.054 \\
\hline Total power & $1.000(1.000-1.000)$ & $0.031^{*}$ & $1.000(1.000-1.000)$ & $0.021^{*}$ & $1.000(1.000-1.000)$ & 0.072 \\
\hline VLF power & $1.000(1.000-1.000)$ & 0.132 & $1.000(1.000-1.000)$ & 0.070 & $1.000(1.000-1.000)$ & 0.133 \\
\hline LF power & $1.000(1.000-1.000)$ & 0.077 & $1.000(1.000-1.000)$ & $0.023^{*}$ & $1.000(1.000-1.000)$ & 0.063 \\
\hline HF power & $1.000(0.999-1.000)$ & $0.002^{*}$ & $1.000(1.000-1.000)$ & $0.014^{*}$ & $1.000(1.000-1.000)$ & 0.074 \\
\hline LF power norm & $1.001(0.994-1.009)$ & 0.738 & $0.999(0.99-1.007)$ & 0.733 & $0.994(0.985-1.004)$ & 0.248 \\
\hline HF power norm & $0.999(0.991-1.007)$ & 0.738 & $1.001(0.993-1.01)$ & 0.733 & $1.006(0.996-1.015)$ & 0.248 \\
\hline LF/HF & $1.034(0.959-1.116)$ & 0.381 & $1.014(0.964-1.066)$ & 0.592 & $1.001(0.973-1.031)$ & 0.923 \\
\hline Poincaré SD1 & $0.986(0.975-0.997)$ & $0.010^{*}$ & 0.988 (0.979-0.997) & $0.011^{*}$ & $0.991(0.983-0.999)$ & $0.029^{*}$ \\
\hline Poincaré SD2 & $0.995(0.990-1.000)$ & $0.032^{*}$ & 0.997 (0.994-1.000) & $0.032^{*}$ & $0.998(0.996-1.000)$ & 0.063 \\
\hline SampEn & $0.813(0.604-1.095)$ & 0.173 & $0.730(0.545-0.977)$ & $0.035^{*}$ & $1.000(1.000-1.000)$ & 0.932 \\
\hline ApEn & $1.645(0.752-3.598)$ & 0.213 & $2.319(1.003-5.357)$ & $0.049^{*}$ & $1.241(0.463-3.327)$ & 0.667 \\
\hline DFA, a1 & $0.953(0.585-1.552)$ & 0.846 & $1.031(0.611-1.741)$ & 0.908 & $0.968(0.560-1.672)$ & 0.907 \\
\hline \multirow[t]{3}{*}{ DFA, a2 } & $1.532(0.773-3.034)$ & 0.221 & $1.202(0.782-1.848)$ & 0.401 & $1.184(0.934-1.500)$ & 0.163 \\
\hline & $\mathrm{HR}_{2} \mathrm{~V}_{1}$ & & $\mathrm{HR}_{3} \mathrm{~V}_{1}$ & & $\mathrm{HR}_{3} \mathrm{~V}_{2}$ & \\
\hline & OR $(95 \% \mathrm{Cl})$ & $p$ & OR $(95 \% \mathrm{Cl})$ & $p$ & OR $(95 \% \mathrm{Cl})$ & $p$ \\
\hline Mean NN & $0.999(0.999-1.000)$ & $0.023^{*}$ & $1.000(0.999-1.000)$ & $0.023^{*}$ & $1.000(0.999-1.000)$ & $0.023^{*}$ \\
\hline SDNN & $0.996(0.993-1.000)$ & $0.034^{*}$ & $0.997(0.995-1.000)$ & $0.042^{*}$ & $0.997(0.995-1.000)$ & $0.034^{*}$ \\
\hline RMSSD & $0.991(0.984-0.998)$ & $0.010^{*}$ & $0.992(0.986-0.999)$ & $0.016^{*}$ & $0.993(0.986-0.999)$ & $0.016^{*}$ \\
\hline Skewness & $1.061(0.980-1.149)$ & 0.144 & $1.072(0.978-1.176)$ & 0.139 & $1.098(0.982-1.227)$ & 0.100 \\
\hline Kurtosis & 1.007 (0.999-1.015) & 0.082 & $1.006(0.994-1.017)$ & 0.333 & $1.010(0.995-1.025)$ & 0.195 \\
\hline Triangular index & $0.981(0.958-1.005)$ & 0.119 & $0.982(0.963-1.001)$ & 0.065 & 0.974 (0.949-0.999) & $0.040^{*}$ \\
\hline NN50 & $0.995(0.991-0.999)$ & $0.018^{*}$ & $0.996(0.993-1.000)$ & 0.052 & $0.992(0.985-0.999)$ & $0.035^{*}$ \\
\hline pNN50 & $0.984(0.972-0.997)$ & $0.020^{*}$ & $0.988(0.977-1.000)$ & $0.049^{*}$ & $0.988(0.976-0.999)$ & $0.035^{*}$ \\
\hline NN50n & $0.989(0.979-1.000)$ & $0.043^{*}$ & $0.982(0.964-1.000)$ & 0.054 & $0.974(0.943-1.007)$ & 0.118 \\
\hline pNN50n & 0.969 (0.939-0.999) & $0.046^{*}$ & $0.947(0.895-1.002)$ & 0.058 & $0.960(0.914-1.009)$ & 0.109 \\
\hline Total power & $1.000(1.000-1.000)$ & $0.048^{*}$ & $1.000(1.000-1.000)$ & 0.072 & $1.000(1.000-1.000)$ & $0.029^{*}$ \\
\hline VLF power & $1.000(1.000-1.000)$ & 0.139 & $1.000(1.000-1.000)$ & 0.145 & $1.000(1.000-1.000)$ & 0.074 \\
\hline LF power & $1.000(1.000-1.000)$ & 0.084 & $1.000(1.000-1.000)$ & 0.092 & $1.000(1.000-1.000)$ & $0.027^{*}$ \\
\hline HF power & $1.000(1.000-1.000)$ & $0.005^{*}$ & $1.000(1.000-1.000)$ & $0.010^{*}$ & $1.000(1.000-1.000)$ & $0.022^{*}$ \\
\hline LF power norm & $1.000(0.991-1.008)$ & 0.937 & $0.995(0.985-1.006)$ & 0.382 & $0.995(0.986-1.005)$ & 0.356 \\
\hline HF power norm & $1.000(0.992-1.009)$ & 0.937 & $1.005(0.994-1.015)$ & 0.382 & $1.005(0.995-1.015)$ & 0.356 \\
\hline LF/HF & $1.024(0.970-1.080)$ & 0.387 & $1.003(0.973-1.033)$ & 0.863 & $0.999(0.971-1.029)$ & 0.966 \\
\hline Poincaré SD1 & $0.987(0.978-0.997)$ & $0.010^{*}$ & 0.989 (0.980-0.998) & $0.016^{*}$ & 0.989 (0.981-0.998) & $0.016^{*}$ \\
\hline
\end{tabular}


Table 3 Univariable analysis of $\mathrm{HR}_{n} \mathrm{~V}$ and $\mathrm{HR}_{n} \mathrm{~V}_{m}$ parameters (Continued)

\begin{tabular}{lllllll}
\hline Poincaré SD2 & $0.997(0.995-1.000)$ & $0.039^{*}$ & $0.998(0.996-1.000)$ & $0.045^{*}$ & $0.998(0.996-1.000)$ & $0.037^{*}$ \\
SampEn & $0.854(0.623-1.171)$ & 0.328 & $0.802(0.553-1.161)$ & 0.242 & $0.709(0.500-1.005)$ & 0.053 \\
ApEn & $2.065(0.842-5.064)$ & 0.113 & $1.207(0.499-2.922)$ & 0.677 & $2.558(0.906-7.222)$ & 0.076 \\
DFA, a1 & $0.888(0.514-1.537)$ & 0.672 & $1.039(0.547-1.971)$ & 0.907 & $1.004(0.549-1.835)$ & 0.991 \\
DFA, a2 & $1.557(0.782-3.098)$ & 0.208 & $1.554(0.780-3.093)$ & 0.210 & $1.169(0.764-1.789)$ & 0.472 \\
\hline
\end{tabular}

HRV Heart rate variability, OR Odds ratio, CI Confidence interval, mean NN Average of R-R intervals, SDNN Standard deviation of R-R intervals, RMSSD Square root of the mean squared differences between R-R intervals, NN50 The number of times that the absolute difference between 2 successive R-R intervals exceeds 50 ms, pNN50, NN50 divided by the total number of R-R intervals; NN50n, the number of times that the absolute difference between 2 successive RR ${ }_{n} / R_{n} I_{m}$ sequences exceeds $50 \times n$ ms; pNN50n, NN50n divided by the total number of $R_{n} l / R_{n} I_{m}$ sequences; VLF Very low frequency, $L F$ Low frequency, $H F$ High frequency, $S D$ Standard deviation, SampEn Sample entropy, ApEn Approximate entropy, DFA Detrended fluctuation analysis $* p<0.05$

$\mathrm{RR}_{n} \mathrm{I}_{m}$ sequences. $\mathrm{HRnV}$ was not developed to replace the conventional HRV but to augment it. It enables the creation of additional parameters from raw ECGs, and thus empowers the extraction of supplementary information.

In our clinical case study, we investigated the predictive value of $\mathrm{HRnV}$ parameters in assessing the risk of 30-day MACE for chest pain patients in the ED. In addition to $21 \mathrm{HRV}$ parameters, $115 \mathrm{HRnV}$ parameters were derived, of which 48 were found to be statistically significant in their association with the primary outcome. Notably, even with a small $n$ (three in our study), newly generated $\mathrm{HRnV}$ parameters greatly boosted the number of candidate predictors. When longer ECG records are available, more $\mathrm{HRnV}$ parameters can be calculated. With HRnV parameters, HRV parameters, vital signs, and several established risk factors, we conducted multivariable logistic regression analysis and selected age, diastolic BP, pain score, ST-elevation, STdepression, Q wave, cardiac history, troponin, HRV NN50, and seven HRnV parameters. In addition to traditional risk factors such as $\mathrm{ST}$ segment changes, $\mathrm{HR}_{2} \mathrm{~V}$ ApEn, $H_{2} \mathrm{~V}_{1}$ ApEn, and $\mathrm{HR}_{3} \mathrm{~V}$ skewness were found to be strong predictors for 30-day MACE. Compared to the HEART, TIMI, and GRACE scores, the HRnV model achieved the highest AUC, specificity, PPV, and NPV values at the optimal cut-off points in ROC analysis. This demonstrated the clinical utility of $\mathrm{HRnV}$ in determining the risk of 30-day MACE for ED patients with chest pain.

Due to the wide differential diagnosis for chest pain, accurate stratification is vital, particularly for preventing low-risk patients from obtaining expensive and unnecessary medical testing and intervention [3]. Although the TIMI and GRACE scores have been validated for risk prediction of patients with chest pain in the ED $[4,33$, 34], some criteria used in these scores may be inappropriate for undifferentiated chest pain cohorts in the ED, as they were originally developed for post-acute myocardial infarction patients [1]. In comparison, the HEART score was derived from a population of ED patients with chest pain, and has been extensively validated worldwide
[10, 13, 27, 35]. It has demonstrated its utility in identifying both low-risk patients for possible early discharge and high-risk patients for urgent intervention. Built upon established scores, several chest pain pathways [14, 36-38] have been implemented and tested, particularly for the management of low-risk patients. Than et al. [38] evaluated a TIMI score-based accelerated diagnostic protocol (ADP) with a reported sensitivity of $99.3 \%$ and NPV of $99.1 \%$. Similarly, a systematic review by Laureano-Phillips et al. [39] reported that the HEART score achieved both sensitivity and NPV of 100\% in several validation studies. Furthermore, a cost-effectiveness study conducted in Brisbane, Australia reported economic benefits by adopting an ADP in the ED, with reduction in expected cost and length of stay amongst patients with chest pain [40].

Most established clinical scores use conventional risk factors such as biomarkers, medical history, and presenting vital signs. However, patient history can sometimes be subjective and blood tests, such as troponin, require waiting time. HRV, as a noninvasive measure, can be easily calculated from ECGs; it is an objective tool to assess the activities of the ANS [19]. It also has the advantage of requiring only several minutes to acquire (five to six minutes in our protocol), which is much faster than serum biomarkers. Over the past decades, HRV has been widely investigated in a broad range of clinical applications, particularly in cardiovascular research. Apart from being associated with sudden cardiac death [18], HRV also showed significant correlations with adverse clinical outcomes in prehospital setting [41] and with MACE outcomes in ED patients with chest pain [17]. HRV parameters have been integrated with other risk factors into machine learning algorithms to predict adverse outcomes $[42,43]$. These promising results motivated the use of HRV to develop objective and computerized risk stratification tools for chest pain patients $[44,45]$. In an updated review of clinical scores for chest pain, Liu et al. [5] summarized several studies which aimed to develop alternative techniques for risk stratification.

This study aimed to present novel HRnV representation and its measures and investigate their association 
Table 4 Multivariable analysis of $H R_{n} V$ and $H R_{n} V_{m}$ parameters by adjusting for age and sex

\begin{tabular}{|c|c|c|c|c|c|c|}
\hline & HRV & & $\mathrm{HR}_{2} \mathrm{~V}$ & & $\mathrm{HR}_{3} \mathrm{~V}$ & \\
\hline & OR $(95 \% \mathrm{Cl})$ & $\mathrm{p}$ & OR $(95 \% \mathrm{Cl})$ & $\mathrm{p}$ & OR $(95 \% \mathrm{Cl})$ & $\mathrm{p}$ \\
\hline Mean NN & $0.999(0.998-1)$ & $0.005^{*}$ & $0.999(0.999-1.000)$ & $0.005^{*}$ & 1.000 (0.999-1.000) & $0.005^{*}$ \\
\hline SDNN & $0.993(0.986-0.999)$ & $0.035^{*}$ & $0.996(0.992-1.000)$ & $0.040^{*}$ & $0.998(0.995-1.000)$ & 0.093 \\
\hline RMSSD & $0.990(0.982-0.998)$ & $0.011^{*}$ & $0.992(0.985-0.999)$ & $0.016^{*}$ & $0.994(0.988-1.000)$ & $0.047^{*}$ \\
\hline Skewness & $1.064(0.995-1.138)$ & 0.068 & $1.082(0.983-1.191)$ & 0.109 & $1.140(1.005-1.293)$ & $0.042^{*}$ \\
\hline Kurtosis & $1.005(1.000-1.011)$ & $0.047^{*}$ & $1.008(0.997-1.019)$ & 0.139 & $1.011(0.993-1.030)$ & 0.238 \\
\hline Triangular index & $0.967(0.93-1.006)$ & 0.093 & $0.971(0.940-1.002)$ & 0.070 & $0.982(0.953-1.013)$ & 0.256 \\
\hline NN50 & $0.993(0.988-0.999)$ & $0.013^{*}$ & $0.991(0.982-0.999)$ & $0.030^{*}$ & $0.990(0.979-1.001)$ & 0.078 \\
\hline pNN50 & $0.979(0.963-0.996)$ & $0.015^{*}$ & $0.986(0.972-0.999)$ & $0.033^{*}$ & $0.989(0.977-1.001)$ & 0.063 \\
\hline NN50n & - & - & $0.983(0.964-1.002)$ & 0.081 & $0.954(0.906-1.005)$ & 0.077 \\
\hline pNN50n & - & - & $0.975(0.947-1.004)$ & 0.086 & $0.952(0.903-1.004)$ & 0.069 \\
\hline Total power & $1.000(1.000-1.000)$ & $0.042^{*}$ & $1.000(1.000-1.000)$ & $0.026^{*}$ & $1.000(1.000-1.000)$ & 0.104 \\
\hline VLF power & $1.000(1.000-1.000)$ & 0.167 & $1.000(1.000-1.000)$ & 0.082 & $1.000(1.000-1.000)$ & 0.152 \\
\hline LF power & $1.000(1.000-1.000)$ & 0.093 & $1.000(1.000-1.000)$ & $0.033^{*}$ & $1.000(1.000-1.000)$ & 0.105 \\
\hline HF power & $1.000(0.999-1.000)$ & $0.003^{*}$ & $1.000(1.000-1.000)$ & $0.016^{*}$ & $1.000(1.000-1.000)$ & 0.101 \\
\hline LF power norm & $1.002(0.994-1.011)$ & 0.589 & 0.999 (0.990-1.007) & 0.769 & $0.994(0.984-1.003)$ & 0.202 \\
\hline HF power norm & 0.998 (0.989-1.006) & 0.589 & $1.001(0.993-1.010)$ & 0.769 & $1.006(0.997-1.016)$ & 0.202 \\
\hline $\mathrm{LF} / \mathrm{HF}$ & $1.039(0.961-1.124)$ & 0.336 & $1.013(0.962-1.066)$ & 0.620 & 0.999 (0.970-1.028) & 0.928 \\
\hline Poincaré SD1 & $0.986(0.975-0.997)$ & $0.011^{*}$ & 0.989 (0.980-0.998) & $0.016^{*}$ & $0.992(0.983-1.000)$ & $0.047^{*}$ \\
\hline Poincaré SD2 & $0.995(0.990-1.000)$ & $0.050^{*}$ & $0.997(0.994-1.000)$ & $0.046^{*}$ & $0.998(0.996-1.000)$ & 0.098 \\
\hline SampEn & $0.852(0.630-1.152)$ & 0.297 & $0.752(0.559-1.010)$ & 0.058 & $1.000(1.000-1.000)$ & 0.956 \\
\hline ApEn & 1.669 (0.754-3.693) & 0.207 & 2.668 (1.139-6.246) & $0.024^{*}$ & 1.507 (0.555-4.096) & 0.421 \\
\hline DFA, a1 & $0.991(0.593-1.654)$ & 0.971 & $1.072(0.622-1.848)$ & 0.802 & $0.962(0.550-1.682)$ & 0.891 \\
\hline \multirow[t]{3}{*}{ DFA, a2 } & $1.499(0.750-2.993)$ & 0.252 & $1.204(0.782-1.853)$ & 0.400 & $1.193(0.941-1.512)$ & 0.146 \\
\hline & $\mathrm{HR}_{2} \mathrm{~V}_{1}$ & & $\mathrm{HR}_{3} \mathrm{~V}_{1}$ & & $\mathrm{HR}_{3} \mathrm{~V}_{2}$ & \\
\hline & OR $(95 \% \mathrm{Cl})$ & $\mathrm{p}$ & OR $(95 \% \mathrm{Cl})$ & $\mathrm{p}$ & OR $(95 \% \mathrm{Cl})$ & $p$ \\
\hline Mean NN & 0.999 (0.999-1.000) & $0.005^{*}$ & $1.000(0.999-1.000)$ & $0.005^{*}$ & $1.000(0.999-1.000)$ & $0.005^{*}$ \\
\hline SDNN & $0.996(0.993-1.000)$ & 0.052 & $0.998(0.995-1.000)$ & 0.064 & $0.997(0.995-1.000)$ & $0.049^{*}$ \\
\hline RMSSD & $0.992(0.985-0.998)$ & $0.015^{*}$ & $0.993(0.986-0.999)$ & $0.023^{*}$ & 0.993 (0.987-0.999) & $0.023^{*}$ \\
\hline Skewness & 1.066 (0.984-1.156) & 0.118 & $1.079(0.983-1.185)$ & 0.108 & 1.099 (0.982-1.229) & 0.099 \\
\hline Kurtosis & 1.007 (0.999-1.015) & 0.096 & $1.005(0.994-1.017)$ & 0.377 & $1.010(0.994-1.025)$ & 0.218 \\
\hline Triangular index & $0.985(0.960-1.010)$ & 0.234 & $0.985(0.965-1.005)$ & 0.137 & $0.977(0.951-1.003)$ & 0.088 \\
\hline NN50 & $0.996(0.991-1.000)$ & $0.047^{*}$ & $0.997(0.993-1.001)$ & 0.130 & $0.993(0.986-1.001)$ & 0.084 \\
\hline pNN50 & $0.986(0.973-1.000)$ & $0.046^{*}$ & $0.990(0.979-1.002)$ & 0.111 & $0.989(0.978-1.001)$ & 0.076 \\
\hline NN50n & $0.990(0.980-1.000)$ & $0.059^{*}$ & $0.982(0.963-1.001)$ & 0.064 & $0.975(0.943-1.008)$ & 0.142 \\
\hline pNN50n & $0.971(0.941-1.002)$ & 0.063 & $0.947(0.893-1.004)$ & 0.067 & $0.962(0.915-1.012)$ & 0.131 \\
\hline Total power & $1.000(1.000-1.000)$ & 0.064 & $1.000(1.000-1.000)$ & 0.096 & $1.000(1.000-1.000)$ & $0.035^{*}$ \\
\hline VLF power & $1.000(1.000-1.000)$ & 0.173 & $1.000(1.000-1.000)$ & 0.180 & $1.000(1.000-1.000)$ & 0.086 \\
\hline LF power & $1.000(1.000-1.000)$ & 0.100 & $1.000(1.000-1.000)$ & 0.108 & $1.000(1.000-1.000)$ & $0.037^{*}$ \\
\hline HF power & $1.000(1.000-1.000)$ & $0.006^{*}$ & $1.000(1.000-1.000)$ & $0.014^{*}$ & $1.000(1.000-1.000)$ & $0.025^{*}$ \\
\hline LF power norm & $1.000(0.991-1.009)$ & 0.960 & $0.995(0.984-1.005)$ & 0.324 & $0.995(0.985-1.005)$ & 0.329 \\
\hline HF power norm & $1.000(0.991-1.009)$ & 0.960 & $1.005(0.995-1.016)$ & 0.324 & $1.005(0.995-1.015)$ & 0.329 \\
\hline LF/HF & $1.023(0.968-1.081)$ & 0.428 & $0.999(0.969-1.030)$ & 0.940 & $0.996(0.967-1.026)$ & 0.786 \\
\hline Poincaré SD1 & $0.988(0.979-0.998)$ & $0.015^{*}$ & $0.990(0.981-0.999)$ & $0.023^{*}$ & 0.990 (0.981-0.999) & $0.023^{*}$ \\
\hline
\end{tabular}


Table 4 Multivariable analysis of $\mathrm{HR}_{n} \mathrm{~V}$ and $\mathrm{HR}_{n} \mathrm{~V}_{m}$ parameters by adjusting for age and sex (Continued)

\begin{tabular}{lllllll}
\hline Poincaré SD2 & $0.997(0.995-1.000)$ & 0.059 & $0.998(0.997-1.000)$ & 0.068 & $0.998(0.996-1.000)$ & 0.052 \\
SampEn & $0.870(0.632-1.197)$ & 0.393 & $0.842(0.578-1.227)$ & 0.371 & $0.716(0.504-1.019)$ & 0.064 \\
ApEn & $2.520(1.009-6.298)$ & $0.048^{*}$ & $1.413(0.575-3.471)$ & 0.451 & $3.461(1.201-9.971)$ & $0.021^{*}$ \\
DFA, a1 & $0.898(0.508-1.587)$ & 0.710 & $1.068(0.555-2.058)$ & 0.843 & $1.005(0.543-1.838)$ & 0.988 \\
DFA, a2 & $1.507(0.751-3.025)$ & 0.249 & $1.500(0.746-3.014)$ & 0.255 & $1.172(0.764-1.798)$ & 0.467 \\
\hline
\end{tabular}

HRV Heart rate variability, OR Odds ratio, CI Confidence interval, mean NN average of R-R intervals, SDNN Standard deviation of R-R intervals, RMSSD Square root of the mean squared differences between R-R intervals, NN50, the number of times that the absolute difference between 2 successive R-R intervals exceeds 50 ms; pNN50, NN50 divided by the total number of R-R intervals; NN50n, the number of times that the absolute difference between 2 successive $R R_{n} l / R R_{n} I_{m}$ sequences exceeds $50 \times n$ ms; pNN50n, NN50n divided by the total number of $R_{n} 1 / R_{n} l_{m}$ sequences; VLF Very low frequency, LF Low frequency, $H F$ High frequency, $S D$ Standard deviation, SampEn Sample entropy, ApEn Approximate entropy, DFA Detrended fluctuation analysis ${ }^{*} p<0.05$

with clinical outcomes. Although HRnV parameters showed promising performance in identifying high-risk chest pain patients, this study was not intended to create a ready-to-use clinical tool. Instead, we demonstrated the feasibility of utilizing HRnV parameters to augment conventional HRV and risk factors in designing a prediction tool/score. These $\mathrm{HRnV}$ parameters can be readily calculated without the collection of supplementary data. In this study, with five to six-minute ECG recording and $n=3$, five-fold more $\mathrm{HRnV}$ parameters were calculated compared to HRV alone. When longer ECG recordings

Table 5 Multivariable analysis with stepwise logistic regression (backward selection) on all variables

\begin{tabular}{|c|c|c|}
\hline Variable & Adjusted OR & $95 \% \mathrm{Cl}$ \\
\hline Age & 1.021 & $1.002-1.041$ \\
\hline Diastolic BP & 1.018 & $1.003-1.034$ \\
\hline Pain score & 1.082 & $1.003-1.168$ \\
\hline ST-elevation & 6.449 & $2.762-15.059$ \\
\hline ST-depression & 4.827 & $2.511-9.277$ \\
\hline Q wave & 3.383 & $1.668-6.860$ \\
\hline Cardiac history ${ }^{a}$ & 7.838 & $5.192-11.832$ \\
\hline Troponin & 4.406 & $3.218-6.033$ \\
\hline HRV NN50 & 0.981 & $0.970-0.991$ \\
\hline $\mathrm{HR}_{2} \mathrm{~V}$ skewness & 0.806 & $0.622-1.045$ \\
\hline $\mathrm{HR}_{2}$ V SampEn & 0.600 & $0.348-1.035$ \\
\hline $\mathrm{HR}_{2} \vee \mathrm{ApEn}$ & 0.095 & $0.014-0.628$ \\
\hline $\mathrm{HR}_{2} \mathrm{~V}_{1}$ ApEn & 19.700 & $2.942-131.900$ \\
\hline $\mathrm{HR}_{3} \mathrm{~V}$ RMSSD & 1.024 & $1.008-1.040$ \\
\hline $\mathrm{HR}_{3} \mathrm{~V}$ skewness & 1.560 & $1.116-2.181$ \\
\hline $\mathrm{HR}_{3} \mathrm{~V}_{2} \mathrm{HF}$ power & 1.000 & $1.000-1.000$ \\
\hline
\end{tabular}

BP Blood pressure, HRV Heart rate variability, OR Odds ratio, CI Confidence interval; mean NN, average of R-R intervals; RMSSD, square root of the mean squared differences between R-R intervals; NN50, the number of times that the absolute difference between 2 successive R-R intervals exceeds $50 \mathrm{~ms}$; $L F$ Low frequency, HF High frequency, SampEn Sample entropy, ApEn Approximate entropy

${ }^{a}$ Cardiac history was a numeric value that was derived from the narrative in the hospital charts. Its value was zero if the patient history contained characteristics of atypical cardiac chest pain; Its value was two if the history contained characteristics of typical cardiac chest pain; Its value was one if the history contained characteristics of both atypical and typical cardiac chest pain are available and parameter $n$ is larger, more HRnV parameters can be derived. To build a HRnV-based risk stratification tool, a systematic approach is needed to derive a point-based, consistent score to ease its clinical application and practical implementation.

As a natural extension of conventional HRV, HRnV representation creates the opportunity to generate additional parameters. This representation could also serve as a smoother for RRIs, making them less sensitive to sudden changes caused by abnormal heart beats (e.g. very short or very long RRI). However, since $H R n V$ is a novel representation of beat-to-beat variations in ECG, many technical issues need to be addressed in future research. For instance, as shown in Table 2, SampEn became larger when the available number of data points was less than 200 [19], suggesting that additional research is required to investigate its applicability to short ECG records. Moreover, parameters NN50n and pNN50n are newly introduced in HRnV representation only. They characterize the number of times that the absolute difference between two successive $\mathrm{RR}_{n} \mathrm{I}$ sequences exceeds $50 \times n \mathrm{~ms}$, by assuming that the absolute difference may be magnified when the corresponding $R_{n} I$ is $n$ times longer than RRI. Thus, in-depth investigations are required in the selection of appropriate thresholds. More importantly, physiological interpretations of the HRnV parameters and their normal values [29] need to be determined through numerous research. One example is the identification of frequency bands that correlate with certain physiological phenomenon. In the current analysis, the conventional cut-off values were adopted (i.e., $\leq 0.04 \mathrm{~Hz}$ as very low frequency range; $0.04-0.15 \mathrm{~Hz}$ as low frequency range; $0.15-0.4 \mathrm{~Hz}$ as high frequency range). With the increase in $n$, frequency domain analysis may need to be changed accordingly.

Beyond its use in risk stratification of ED patients with chest pain, $\mathrm{HRnV}$ can potentially be used in other clinical domains, where conventional HRV has been extensively investigated [46-49]. With the augmented $R_{n} I$ and $\mathrm{RR}_{n} \mathrm{I}_{m}$ sequences, HRnV could possibly capture more dynamic changes in cardiac rhythms than HRV. This capability enables the extraction of additional information 


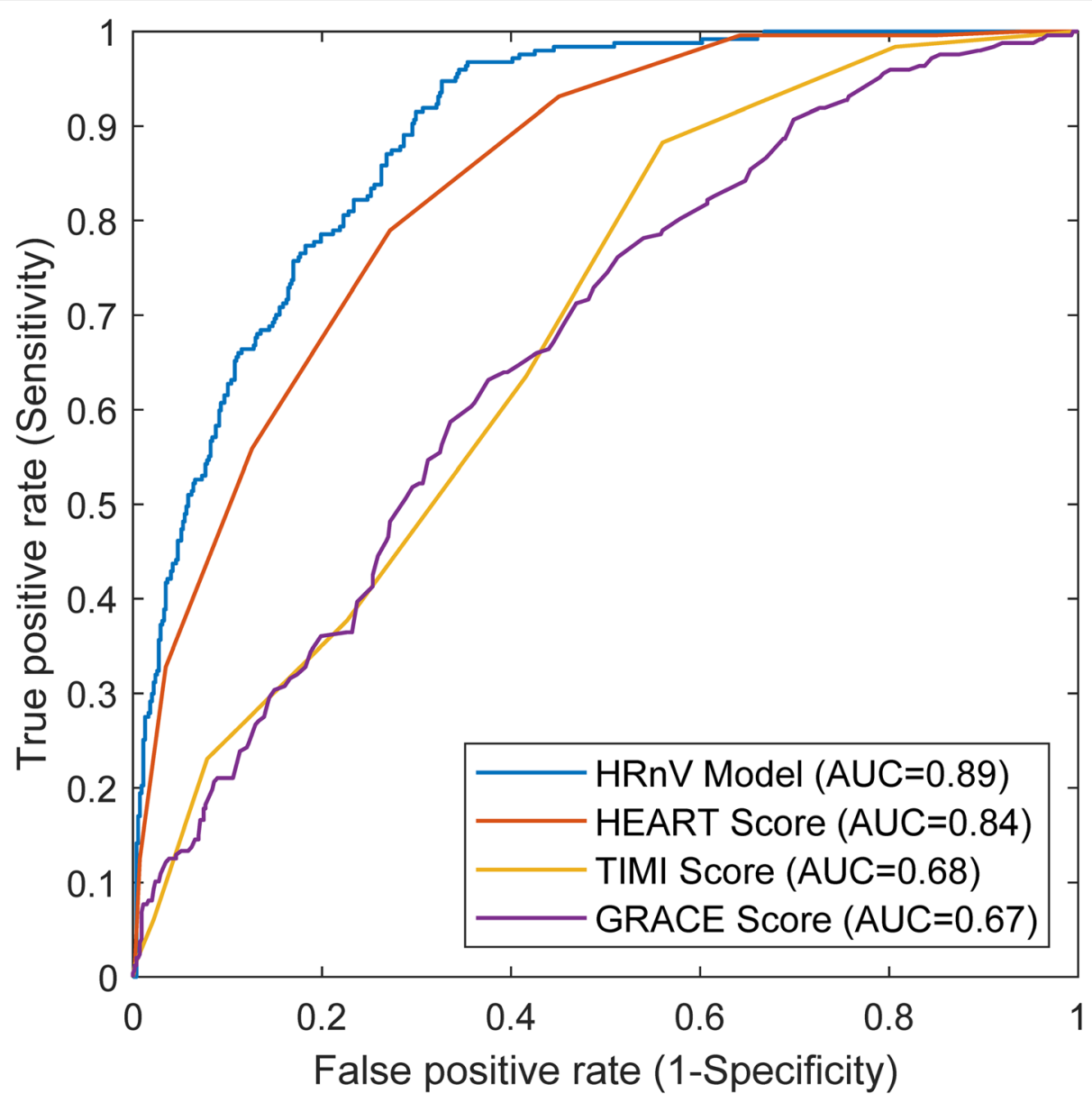

Fig. 2 The receiver operating characteristic (ROC) curves produced by heart rate n-variability (HRnV) model (performance was based on leaveone-out cross-validation), the History, ECG, Age, Risk factors and Troponin (HEART) score, the Thrombolysis in Myocardial Infarction (TIMI) score, and the Global Registry of Acute Coronary Events (GRACE) score

Table 6 Comparison of performance of the HRnV model (based on leave-one-out cross-validation), HEART, TIMI, and GRACE sCores in predicting 30-day major adverse cardiac events (MACE)

\begin{tabular}{lllllll}
\hline & AUC $(95 \% \mathrm{Cl})$ & Cut-off & Sensitivity $(95 \% \mathrm{Cl})$ & Specificity $(95 \% \mathrm{Cl})$ & PPV (95\% Cl) & $\mathrm{NPV}(95 \% \mathrm{Cl})$ \\
\hline HRnV Model & $0.888(0.860-0.917)$ & $0.3611^{\mathrm{a}}$ & $77.3 \%(72.1-82.5 \%)$ & $81.8 \%(78.5-85.0 \%)$ & $65.6 \%(60.2-71.1 \%)$ & $88.9 \%(86.1-91.6 \%)$ \\
& - & 0.0352 & $99.2 \%(98.1-100.0 \%)$ & $39.6 \%(35.5-43.7 \%)$ & $42.5 \%(38.5-46.6 \%)$ & $99.1 \%(97.8-100.0 \%)$ \\
\multirow{2}{*}{ HEART } & $0.841(0.808-0.874)$ & $5^{\mathrm{a}}$ & $78.9 \%(73.9-84.0 \%)$ & $72.8 \%(69.1-76.5 \%)$ & $56.7 \%(51.4-61.9 \%)$ & $88.5 \%(85.5-91.4 \%)$ \\
& - & 3 & $99.6 \%(98.8-100.0 \%)$ & $35.8 \%(31.8-39.8 \%)$ & $41.1 \%(37.2-45.1 \%)$ & $99.5 \%(98.5-100.0 \%)$ \\
\multirow{2}{*}{ TIMI } & $0.681(0.639-0.723)$ & $2^{\mathrm{a}}$ & $63.6 \%(57.6-69.6 \%)$ & $58.4 \%(54.3-62.5 \%)$ & $40.8 \%(35.9-45.7 \%)$ & $78.0 \%(74.0-82.1 \%)$ \\
& - & 0 & $98.4 \%(96.8-100.0 \%)$ & $19.3 \%(16.0-22.7 \%)$ & $35.5 \%(31.9-39.1 \%)$ & $96.4 \%(92.9-99.9 \%)$ \\
\multirow{2}{*}{ GRACE } & $0.665(0.623-0.707)$ & $107^{\mathrm{a}}$ & $64.0 \%(58.0-70.0 \%)$ & $60.8 \%(56.7-64.9 \%)$ & $42.4 \%(37.3-47.4 \%)$ & $78.9 \%(75.0-82.8 \%)$ \\
& - & 60 & $98.8 \%(97.4-100.0 \%)$ & $8.0 \%(5.8-10.3 \%)$ & $32.6 \%(29.3-36.0 \%)$ & $93.6 \%(86.6-100.0 \%)$ \\
\hline
\end{tabular}

AUC Area under the curve, Cl Confidence interval, PPV Positive predictive value, NPV Negative predictive value, HEART History, ECG, Age, Risk factors and Troponin, TIMI Thrombolysis in Myocardial Infarction, GRACE Global Registry of Acute Coronary Events

${ }^{a}$ Optimal cut-off values, defined as the points nearest to the upper-left corner on the ROC curves 
from limited raw ECGs. This study utilized HRnV parameters as independent risk factors and analyzed them with traditional biostatistical methods. There are multiple ways to use HRnV parameters, e.g. each set of HRnV parameters can be analyzed individually and subsequently combined with an ensemble learning [50] (a special type of machine learning algorithm) architecture to reach a decision. However, artificial intelligence and machine learning methods generally create black-box predictive models, making interpretation a challenge [51].

\section{Limitations}

This study has several limitations. First, we did not develop a scoring tool for practical clinical use. The primary aim of this study was to demonstrate the feasibility of using HRnV parameters and common risk factors to build predictive models. Second, the HRnV model was evaluated with LOOCV strategy due to the small sample size. Ideally, separate patient cohorts are needed to train and test prediction models. When a new scoring tool is developed, it is necessary to conduct external validations on cohorts with diverse patient characteristics. Furthermore, properly designed clinical pathways are needed as well. Third, the patients included in this study were mainly from the high acuity group, resulting in a higher 30-day MACE rate (i.e., 31\%) compared to other similar studies $[10,39]$. As a result, the generalizability of the HRnV model developed in this study may be uncertain in other patient cohorts. Fourth, the calculated HRnV and HRV parameters depended on the choice of tools and methods for ECG signal analysis. Thus, the values of these parameters may vary across studies. Last, the physiology of $\mathrm{HRnV}$ and interpretations of its measures are mostly unknown; calculation of some parameters also needs to be standardized. All these require future collaborative research efforts between clinicians and scientists to address.

\section{Conclusions}

In this study, we proposed a novel HRnV representation and investigated the use of $\mathrm{HRnV}$ and established risk factors to develop a predictive model for risk stratification of patients with chest pain in the ED. Multiple HRnV parameters were found to be statistically significant predictors, which effectively augmented conventional HRV, vital signs, troponin, and cardiac risk factors in building an effective model with good discrimination performance. The HRnV model outperformed the HEART, TIMI, and GRACE scores in the ROC analysis. It also demonstrated its capability in identifying low-risk patients, which could potentially be used to build a new clinical pathway. Moving forward, we suggest further development of a point-based, ready-to-use $\mathrm{HRnV}$ risk stratification tool. Although some issues remain to be addressed, we hope to stimulate a new stream of research on HRnV. We believe that future endeavors in this field will lead to the possibility of in-depth evaluation of the associations between $\mathrm{HRnV}$ measures and various human diseases.

\section{Abbreviations}

ACS: Acute coronary syndrome; ADP: Accelerated diagnostic protocol; AMI: Acute myocardial infarction; ANS: Autonomic nervous system;

AUC: Area under the curve; ApEn: Approximate entropy; CABG: Coronary artery bypass graft; Cl: Confidence interval; DFA: Detrended fluctuation analysis; ED: Emergency department; EHR: Electronic health records; GRACE: Global registry of acute coronary events; HEART: History, ECG, age, risk factors and troponin; HF: High frequency; HRnV: Heart rate n-variability; HRV: Heart rate variability; LF: Low frequency; LOOCV: Leave-one-out crossvalidation; MACE: Major adverse cardiac events; Mean NN: Average of R-R intervals; MI: Myocardial infarction; NN50: The number of times that the absolute difference between 2 successive R-R intervals exceeds $50 \mathrm{~ms}$; NN50n: The number of times that the absolute difference between 2 successive RR $n / R R_{n} I_{m}$ sequences exceeds $50 \times n$ ms; NPV: Negative predictive value; PACS: Patient acuity category scale; PCl: Percutaneous coronary intervention; pNN50: NN50 divided by the total number of R-R intervals; pNN50n: NN50n divided by the total number of $R_{n} I / R_{n} I_{m}$ sequences; PPV: Positive predictive value; RMSSD: Square root of the mean squared differences between R-R intervals; ROC: Receiver operating characteristic; RRI: R-R interval; SampEn: Sample entropy; SD: Standard deviation; SDNN: Standard deviation of R-R intervals; STEMI: ST-elevation myocardial infarction; TIMI: Thrombolysis in Myocardial Infarction; VLF: Very low frequency

\section{Acknowledgements}

We would like to thank and acknowledge the contributions of doctors, nurses, and clinical research coordinators from the Department of Emergency Medicine, Singapore General Hospital.

\section{Authors' contributions}

$\mathrm{NL}$ invented the HRnV representation, conceived the study, supervised the project, and wrote the first draft of the manuscript. NL, DG, ZXK, and FX performed the analyses. NL, DG, ZXK, AFWH, FX, TT, JTS, PPP, BC, SHL, JWCT, and $\mathrm{MEHO}$ contributed to evaluation of the HRnV measures, interpretation of the results, and revision of the manuscript. NL, DG, ZXK, AFWH, FX, TT, JTS, PPP, BC, SHL, JWCT, and MEHO approved the final manuscript.

\section{Funding}

This work was supported by the SHF Foundation Research Grant (SHF) FG652P/2017). The study sponsor was not involved in the study design; in the collection, analysis and interpretation of data; in the writing of the manuscript; and in the decision to submit the manuscript for publication.

\section{Availability of data and materials}

The datasets used and/or analyzed during the current study are available from the corresponding author on reasonable request.

\section{Ethics approval and consent to participate}

The ethical approval was obtained from the Centralized Institutional Review Board (CIRB, Ref: 2014/584/C) of SingHealth, in which patient consent was waived.

\section{Consent for publication}

Not applicable.

\section{Competing interests}

$\mathrm{NL}$ and MEHO hold patents related to using heart rate variability and artificial intelligence for medical monitoring. NL, DG, ZXK, and MEHO are currently advisers to TIIM SG. The other authors report no conflicts.

\section{Author details}

${ }^{1}$ Duke-NUS Medical School, National University of Singapore, 8 College Road, Singapore 169857, Singapore. ${ }^{2}$ Health Services Research Centre, Singapore 
Health Services, 20 College Road, Singapore 169856, Singapore. ${ }^{3}$ SingHealth Duke-NUS Emergency Medicine Academic Clinical Programme, Singapore, Singapore. ${ }^{4}$ Department of Emergency Medicine, Singapore General Hospital, Singapore, Singapore. ${ }^{5}$ National Heart Research Institute Singapore, National Heart Centre Singapore, Singapore, Singapore. ${ }^{6}$ Department of Emergency and Critical Care Medicine, Nippon Medical School Musashikosugi Hospital, Tokyo, Japan. ' School of Medicine, Stanford University, Stanford, California, USA. ${ }^{8}$ National Heart Centre Singapore, Singapore, Singapore.

\section{Received: 15 October 2019 Accepted: 30 March 2020} Published online: 10 April 2020

\section{References}

1. Long B, Koyfman A. Best clinical practice: current controversies in the evaluation of Low-risk chest pain with risk stratification aids. Part 2. J Emerg Med. 2017;52(1):43-51.

2. Long B, Koyfman A. Best clinical practice: current controversies in evaluation of Low-risk chest pain-part 1. J Emerg Med. 2016;51(6):668-76.

3. Hollander JE, Than M, Mueller C. State-of-the-art evaluation of emergency department patients presenting with potential acute coronary syndromes. Circulation. 2016;134(7):547-64.

4. Backus BE, Six AJ, Kelder JH, Gibler WB, Moll FL, Doevendans PA. Risk scores for patients with chest pain: evaluation in the emergency department. Curr Cardiol Rev. 2011;7(1):2-8.

5. Liu N, Ng JCJ, Ting CE, Sakamoto JT, Ho AFW, Koh ZX, Pek PP, Lim SH, Ong $\mathrm{MEH}$. Clinical scores for risk stratification of chest pain patients in the emergency department: an updated systematic review. J Emerg Crit Care Med. 2018;2:16.

6. Six AJ, Cullen L, Backus BE, Greenslade J, Parsonage W, Aldous S, Doevendans PA, Than M. The HEART score for the assessment of patients with chest pain in the emergency department: a multinational validation study. Crit Pathw Cardiol. 2013;12(3):121-6.

7. Antman EM, Cohen M, Bernink PJ, McCabe CH, Horacek T, Papuchis $G$, Mautner B, Corbalan R, Radley D, Braunwald E. The TIMI risk score for unstable angina/non-ST elevation Ml: a method for prognostication and therapeutic decision making. Jama. 2000;284(7):835-42.

8. Granger CB, Goldberg RJ, Dabbous O, Pieper KS, Eagle KA, Cannon CP, Van De Werf F, Avezum A, Goodman SG, Flather MD, et al. Predictors of hospital mortality in the global registry of acute coronary events. Arch Intern Med. 2003;163(19):2345-53.

9. Sakamoto JT, Liu N, Koh ZX, Guo D, Heldeweg MLA, Ng JCJ, Ong MEH. Integrating heart rate variability, vital signs, electrocardiogram, and troponin to triage chest pain patients in the ED. Am J Emerg Med. 2018;36(2):185192.

10. Poldervaart JM, Langedijk M, Backus BE, Dekker IMC, Six AJ, Doevendans PA, Hoes AW, Reitsma JB. Comparison of the GRACE, HEART and TIMI score to predict major adverse cardiac events in chest pain patients at the emergency department. Int J Cardiol. 2017;227:656-61.

11. Fernando SM, Tran A, Cheng W, Rochwerg B, Taljaard M, Thiruganasambandamoorthy V, Kyeremanteng K, Perry JJ. Prognostic accuracy of the HEART score for prediction of major adverse cardiac events in patients presenting with chest pain: a systematic review and metaanalysis. Acad Emerg Med Off J Soc Acad Emerg Med. 2019;26(2):140-51.

12. Reaney PDW, Elliott HI, Noman A, Cooper JG. Risk stratifying chest pain patients in the emergency department using HEART, GRACE and TIMI scores, with a single contemporary troponin result, to predict major adverse cardiac events. Emerg Med J. 2018;35(7):420-7.

13. Van Den Berg P, Body R. The HEART score for early rule out of acute coronary syndromes in the emergency department: a systematic review and meta-analysis. Eur Heart J Acute Cardiovasc Care. 2018;7(2):111-9.

14. Mahler SA, Riley RF, Hiestand BC, Russell GB, Hoekstra JW, Lefebvre CW, Nicks BA, Cline DM, Askew KL, Elliott SB, et al. The HEART pathway randomized trial: identifying emergency department patients with acute chest pain for early discharge. Circ Cardiovasc Qual Outcomes. 2015;8(2): $195-203$.

15. Liu N, Lin Z, Cao J, Koh ZX, Zhang T, Huang G-B, Ser W, Ong MEH. An intelligent scoring system and its application to cardiac arrest prediction. IEEE Trans Inf Technol Biomed. 2012;16(6):1324-31.

16. Liu N, Koh ZX, Chua ECP, Tan LML, Lin Z, Mirza B, Ong MEH. Risk scoring for prediction of acute cardiac complications from imbalanced clinical data. IEEE J Biomed Health Inform. 2014;18(6):1894-902.
17. Ong MEH, Goh K, Fook-Chong S, Haaland B, Wai KL, Koh ZX, Shahidah N, Lin Z. Heart rate variability risk score for prediction of acute cardiac complications in ED patients with chest pain. Am J Emerg Med. 2013;31(8): 1201-7.

18. Task Force of the European Society of Cardiology and the North American Society of Pacing and Electrophysiology. Heart rate variability: standards of measurement, physiological interpretation and clinical use. Circulation. 1996; 93(5):1043-65.

19. Rajendra Acharya U, Paul Joseph K, Kannathal N, Lim CM, Suri JS. Heart rate variability: a review. Med Biol Eng Comput. 2006;44(12):1031-51.

20. Billman GE. Heart rate variability - a historical perspective. Front Physiol. 2011;2:86.

21. Carpeggiani C, L'Abbate A, Landi P, Michelassi C, Raciti M, Macerata A, Emdin M. Early assessment of heart rate variability is predictive of in-hospital death and major complications after acute myocardial infarction. Int J Cardiol. 2004;96(3):361-8.

22. Vest AN, Da Poian G, Li Q, Liu C, Nemati S, Shah AJ, Clifford GD. An open source benchmarked toolbox for cardiovascular waveform and interval analysis. Physiol Meas. 2018;39(10):105004.

23. Tarvainen MP, Niskanen JP, Lipponen JA, Ranta-Aho PO, Karjalainen PA. Kubios HRV-heart rate variability analysis software. Comput Methods Prog Biomed. 2014;113:210-20.

24. Liu N, Guo D, Koh ZX, Ho AFW, Ong MEH. Heart rate n-variability (HRnV): A novel representation of beat-to-beat variation in electrocardiogram. bioRxiv. 2018:449504. https://doi.org/10.1101/449504

25. Ho AF, Fook-Chong S, Pek PP, Ng YY, Wong AS, Ong ME. Prehospital presentation of patients with ST-segment elevation myocardial infarction in Singapore. Int J Cardiol. 2013;168(4):4273-6.

26. Januzzi JL Jr, Bamberg F, Lee H, Truong QA, Nichols JH, Karakas M, Mohammed AA, Schlett CL, Nagurney JT, Hoffmann U, et al. High-sensitivity troponin $T$ concentrations in acute chest pain patients evaluated with cardiac computed tomography. Circulation. 2010;121(10):1227-34.

27. Sakamoto JT, Liu N, Koh ZX, Fung NX, Heldeweg ML, Ng JC, Ong ME. Comparing HEART, TIMI, and GRACE scores for prediction of 30-day major adverse cardiac events in high acuity chest pain patients in the emergency department. Int J Cardiol. 2016;221:759-64.

28. Fawcett T. An introduction to ROC analysis. Pattern Recogn Lett. 2006;27(8): 861-74.

29. Shaffer F, Ginsberg JP. An overview of heart rate variability metrics and norms. Front Public Health. 2017;5:258.

30. Peng CK, Havlin S, Stanley HE, Goldberger AL. Quantification of scaling exponents and crossover phenomena in nonstationary heartbeat time series. Chaos. 1995;5(1):82-7.

31. Barbieri R, Matten EC, Alabi AA, Brown EN. A point-process model of human heartbeat intervals: new definitions of heart rate and heart rate variability. Am J Phys Heart Circ Phys. 2005;288(1):H424-35.

32. Vollmer M. A robust, simple and reliable measure of heart rate variability using relative RR intervals. In: Computing in Cardiology Conference (CinC): 6-9 Sept. 2015, vol. 2015; 2015. p. 609-12.

33. Chase M, Robey JL, Zogby KE, Sease KL, Shofer FS, Hollander JE. Prospective validation of the thrombolysis in myocardial infarction risk score in the emergency department chest pain population. Ann Emerg Med. 2006:48(3): 252-9.

34. Pollack CV, Sites FD, Shofer FS, Sease KL, Hollander JE. Application of the TIMI risk score for unstable angina and non-ST elevation acute coronary syndrome to an unselected emergency department chest pain population. Acad Emerg Med Off J Soc Acad Emerg Med. 2006;13(1):13-8.

35. Marcoon S, Chang AM, Lee B, Salhi R, Hollander JE. HEART score to further risk stratify patients with low TIMI scores. Crit Pathw Cardiol. 2013;12(1):1-5.

36. Long B, Oliver J, Streitz M, Koyfman A. An end-user's guide to the HEART score and pathway. Am J Emerg Med. 2017;35(9):1350-5.

37. Than MP, Pickering JW, Aldous SJ, Cullen L, Frampton CM, Peacock WF, Jaffe AS, Goodacre SW, Richards AM, Ardagh MW, et al. Effectiveness of EDACS versus ADAPT accelerated diagnostic pathways for chest pain: a pragmatic randomized controlled trial embedded within practice. Ann Emerg Med. 2016;68(1):93-102 e101.

38. Than M, Cullen L, Reid CM, Lim SH, Aldous S, Ardagh MW, Peacock WF, Parsonage WA, Ho HF, Ko HF, et al. A 2-h diagnostic protocol to assess patients with chest pain symptoms in the Asia-Pacific region (ASPECT): a prospective observational validation study. Lancet (London, England). 2011; 377(9771):1077-84. 
39. Laureano-Phillips J, Robinson RD, Aryal S, Blair S, Wilson D, Boyd K, Schrader $C D$, Zenarosa NR, Wang H. HEART score risk stratification of Low-risk chest pain patients in the emergency department: a systematic review and metaanalysis. Ann Emerg Med. 2019;74(2):187-203.

40. Cheng Q, Greenslade JH, Parsonage WA, Barnett AG, Merollini K, Graves N, Peacock WF, Cullen L. Change to costs and lengths of stay in the emergency department and the Brisbane protocol: an observational study. BMJ Open. 2016;6(2):e009746.

41. Ong ME, Padmanabhan P, Chan YH, Lin Z, Overton J, Ward KR, Fei DY. An observational, prospective study exploring the use of heart rate variability as a predictor of clinical outcomes in pre-hospital ambulance patients. Resuscitation. 2008;78(3):289-97.

42. Liu N, Zhang Z, Ho AFW, Ong MEH. Artificial intelligence in emergency medicine. J Emerg Crit Care Med. 2018;2:82.

43. Chiew CJ, Liu N, Tagami T, Wong TH, Koh ZX, Ong MEH. Heart rate variability based machine learning models for risk prediction of suspected sepsis patients in the emergency department. Medicine. 2019;98(6):e14197.

44. Liu N, Sakamoto JT, Cao J, Koh ZX, Ho AFW, Lin Z, Ong MEH. Ensemblebased risk scoring with extreme learning machine for prediction of adverse cardiac events. Cogn Comput. 2017;9(4):545-54.

45. Liu N, Koh ZX, Goh J, Lin Z, Haaland B, Ting BP, Ong MEH. Prediction of adverse cardiac events in emergency department patients with chest pain using machine learning for variable selection. BMC Med Inform Decis Mak. 2014;14:75.

46. Quintana DS, Alvares GA, Heathers JA. Guidelines for reporting articles on psychiatry and heart rate variability (GRAPH): recommendations to advance research communication. Transl Psychiatry. 2016;6:e803.

47. Kloter E, Barrueto K, Klein SD, Scholkmann F, Wolf U. Heart rate variability as a prognostic factor for Cancer survival - a systematic review. Front Physiol. 2018;9:623.

48. Schroeder EB, Chambless LE, Liao D, Prineas RJ, Evans GW, Rosamond WD, Heiss G. Diabetes, glucose, insulin, and heart rate variability: the atherosclerosis risk in communities (ARIC) study. Diabetes Care. 2005;28(3): 668-74.

49. Buchman TG, Stein PK, Goldstein B. Heart rate variability in critical illness and critical care. Curr Opin Crit Care. 2002;8(4):311-5.

50. Polikar R. Ensemble based systems in decision making. IEEE Circuits Syst Mag. 2006;6(3):21-44.

51. Lundberg SM, Nair B, Vavilala MS, Horibe M, Eisses MJ, Adams T, Liston DE King-Wai Low D, Newman SF, Kim J, et al. Explainable machine-learning predictions for the prevention of hypoxaemia during surgery. Nat Biomed Eng. 2018;2(10):749-60

\section{Publisher's Note}

Springer Nature remains neutral with regard to jurisdictional claims in published maps and institutional affiliations.

Ready to submit your research? Choose BMC and benefit from:

- fast, convenient online submission

- thorough peer review by experienced researchers in your field

- rapid publication on acceptance

- support for research data, including large and complex data types

- gold Open Access which fosters wider collaboration and increased citations

- maximum visibility for your research: over $100 \mathrm{M}$ website views per year

At BMC, research is always in progress.

Learn more biomedcentral.com/submissions 